\title{
Catheter-related blood stream infections in hemodialysis patients
}

\author{
Babak Hadian $^{\mathbb{(}}$, Azita Zafarmohtashami* ${ }^{\mathbb{D}}$, Mahdi Razani ${ }^{\mathbb{D}}$ \\ Department of Nephrology, Lorestan University of Medical Sciences, Khoramabad, Iran
}

\section{A R T I C L E I N F O}

Article Type:

Original

\section{Article History:}

Received: 8 January 2020

Accepted: 20 March 2020

Published online: 23 April 2020

\section{Keywords:}

Hemodialysis,

Catheter-related blood stream

infections,

Vascular access,

Staphylococcus organisms,

End-stage renal disease

\begin{abstract}
A B S T R A C T
Introduction: Proper care of vascular access in hemodialysis patients is important. Catheterrelated bloodstream infection (CRBSI), is a life-threatening complication of hemodialysis. Objectives: Sufficient data about microorganisms and their susceptibility to antibiotics in hemodialysis patients is necessary for handling of CRBSI; therefore, this study performed for better management of patients.

Patients and Methods: All hemodialysis patients from March 2015 to March 2018 who had cultures of catheter and blood samples were studied. Clinical records of 122 patients were reviewed for variables such as catheter and blood culture microorganism types, antibiotic resistance, age, gender, site, comorbidities, and various clinical signs.

Results: Eighty-four cases of catheter cultures were positive for bacteria. Staphylococcus epidermidis was the most common organism (36\%) since Staphylococcus aureus was the second one (28\%). In some cases, multidrug resistant organisms such as Enterobacter baumannii or methicillin-resistant Staphylococcus aureus (MRSA) organisms were grown. Twenty-one percent of $S$. aureus organisms were MRSA. No significant association between important diagnostic data (fever, chills or WBC count) and bacteremia were shown. Gender of patients had a significant statistical association with CRBSI.

Conclusion: Given the necessity of proper management, physicians must empirically initiate antibiotic therapy as soon as possible, until receiving definite culture results, in hemodialysis patients suspected of bacteremia. In our study, both gram-positive and gram-negative organisms were common. Hence, when initial empirical treatment is indicated, the coverage of both gram positive and gram negative organisms must be considered. Vancomycin or other antibiotics that are effective on MRSA must be included in empirical treatment.
\end{abstract}

\section{Implication for health policy/practice/research/medical education}

Catheter-related bloodstream infection (CRBSI) is a critical problem in dialysis center. Given the necessity of prompt management, physicians must empirically initiate antibiotic therapy. Based on this study, coverage of both gram positive and negative organisms must be considered. In our study, male gender was a risk factor for CRBSI.

Please cite this paper as: Hadian B, Zafarmohtashami A, Razani M. Catheter-related blood stream infections in hemodialysis patients. J Renal Inj Prev. 2020; 9(4): 34. doi: 10.34172/jrip.2020.34.

\section{Introduction}

One challenging problem in hemodialysis patients is catheter-related bloodstream infections (CRBSIs). Hemodialysis patients are susceptible to infection due to multiple individual and therapeutic risk factors such as decreased immunity, repeated exposure to the hospital equipment and hemodialysis catheter colonization during hemodialysis sessions (1-3).

In addition, infection is an important factor for hospitalization in hemodialysis patients. CRBSI is a pitfall for health outcomes in these patients. In some centers, approximately $30 \%$ of the hospitalized hemodialysis patients have catheter infections (4).

Hence, proper handling of catheter is necessary for reducing CRBSIs. For decreasing catheter infections, permanent devices were replaced with temporary ones. Still, however, catheter infections and related complications are life-threatening events in dialysis centers. On the other hand, economic burden of infectious events is considerable (5-7).

CRBSIs are mostly common with gram-positive skin flora, particularly staphylococci (1). The most common 
organisms causing CRBSIs are coagulase-negative staphylococci (CoNS), Staphylococcus aureus and enteric gram-negative bacilli, respectively (8). The mature multilayered biofilm that is perhaps 100-fold larger than the microorganisms, results in antibiotic resistance in patients (9).

According to international guidelines, delay in referral of patients in stage 4 of chronic kidney disease (pre-dialysis) to nephrologist and implantation of arteriovenous fistula or graft result in catheter insertion for urgent hemodialysis and increased risk of subsequent complications. Decreased mortality due to infection may be achieved by appropriate choice of antibiotics and avoiding catheter salvage attempts (1).

\section{Objectives}

One important problem is proper management of bacteremia related to hemodialysis catheters. Delay in receiving culture results and lack of characteristic signs and symptoms cause most physicians to initiate empirical antibiotic therapy in patients suspected of CRBSIs. This strategy needs primary information about the epidemiology of CRBSIs in each center.

Therefore, we studied the epidemiology of CRBSIs and related risk factors in hemodialysis centers of Lorestan University of Medical Sciences.

\section{Patients and Methods \\ Study population}

This study was conducted on 122 hemodialysis patients suspected for CRBSIs in hemodialysis centers of Lorestan University of Medical Sciences (March 2015 to March 2018) (10).

\section{Clinical and lab data}

Data collected about the organisms of the catheter line and/or peripheral bloodlines. Antibiotic resistance of the organisms of the catheter and/or blood, age, gender, comorbidities (such as hypertension and diabetes mellitus), site of the catheter and clinical presentation of infection such as fever or chills, leucocyte count and other related characteristics were determined.

\section{Diagnostic methods}

Incidence rates of CRBSI were calculated from pooled data for device and expressed as CRBSI per 1000 catheter - days (method of calculation: the number of days of catheter use divided into episode of bacteremia occurred, multiplied by 1000) (11).

Diagnosis of CRBSIs requires one of the following;

1. Concurrent positive blood cultures of the same organism from the catheter and a peripheral vein.

2. Culture of the same organism from both the catheter tip and at least one percutaneous blood culture.

3. Cultures of the same organism from two peripherally drawn blood cultures. Organisms cultured must meet CRBSI criteria for quantitative blood cultures or differential time to positivity. Additionally, growth of $>15$ colony-forming units (CFU) from a $5-\mathrm{cm}$ segment of the catheter tip by semi-quantitative culture was designated for catheter infection (12).

\section{Patient suspected of CRBSIs}

A case with a dwelled central vein hemodialysis catheter is suspected of CRBSI if patient has clinical characteristics of infection including fever or chills, and/or significant changes in leucocyte counts, without any other obvious sources for infection.

\section{Microbiological methods}

Tips of the catheters were removed by the surgeon and transferred to laboratory in a sterile container, while at least two blood culture samples were taken before removing the catheter (from peripheral vein and catheter line or hub). The antibiotic susceptibility profile for isolated organisms was determined by disk diffusion agar using Müller-Hinton agar according to the Clinical and Laboratory Standards Institute (CLSI) guideline (13).

\section{Ethical issues}

Human rights were respected in accordance with the Helsinki Declaration 1975, as revised in 1983. The ethical committee of Lorestan University of Medical Sciences confirmed the study (ethical code; IR.LUMS. REC.1398.020). The informed consents were taken from the patients. This study was extracted from the M.D, thesis of Mahdi Razani at this University (Thesis\# 1071).

\section{Statistical analysis}

SPSS version 16 was used for statistical analysis. Qualitative variables analyzed by chi-square and quantitative ones by $t$ test, respectively. $P$ value less than 0.05 was considered significant.

\section{Results}

Average age of infected patients was 62.9 years and their gender distribution included 58 men (69\%) and 26 women (31\%), in this study. Our results showed that the incidence of CRBSI was 7.1 episodes per 1000 catheter/days.

About frequency of known etiologic factors for endstage renal disease, hypertension, diabetes mellitus, nephrolithiasis and obstructive uropathy were the most common causes, respectively.

\section{Organisms and antibiotic resistance}

Eighty-four (68\%) of the patients had positive cultures, (bacteria were isolated in eighty-one cultures and fungi in three cultures). The most common organisms were CoNS and Staphylococcus aureus, respectively (Table 1). Microorganisms that, obtained from the catheters or blood 
Table 1. Variety of the organisms in CRBSI

\begin{tabular}{|c|c|c|}
\hline \multicolumn{2}{|c|}{ Organisms } & \multirow{2}{*}{$\frac{\text { Number of organisms (\%) }}{24(28)}$} \\
\hline \multirow{6}{*}{ G+ } & Staphylococcus aureus & \\
\hline & Staphylococcus epidermidis & $31(36)$ \\
\hline & Staphylococcus schleiferi & $3(3.5)$ \\
\hline & Staphylococcus haemolyticus & $2(2)$ \\
\hline & Staphylococcus saprophyticus & $1(1)$ \\
\hline & Enterococcus faecalis & $6(7)$ \\
\hline \multirow{7}{*}{ G- } & Acinetobacter Iwoffii & $1(1)$ \\
\hline & Acinetobacter baumannii & $3(3.5)$ \\
\hline & E. coli & $3(3.5)$ \\
\hline & Enterobacter cloacae & $2(2)$ \\
\hline & Klebsiella pneumoniae & $2(2)$ \\
\hline & Stenotrophomonas maltophilia & $2(2)$ \\
\hline & Pseudomonas aeruginosa & $1(1)$ \\
\hline Fungi & Candida albicans & $3(3.5)$ \\
\hline
\end{tabular}

cultures were gram-positive (Staphylococcus epidermis, Staphylococcus aureus, Staphylococcus schleiferi, Staphylococcus haemolyticus, Staphylococcus saprophyticus, Enterococcus faecalis), gram-negative (Acinetobacter, Escherichia coli, Enterobacter cloacae, Stenotrophomonas maltophilia, Pseudomonas aeruginosa,
Klebsiella pneumonia) and fungi (Candida albicans) (Table 1). According to the antibiotic susceptibility tests from the cultures, the most common antibiotic resistance and most sensitivity in Staphylococcus spp. were reported for penicillin and cefoxitin, respectively. Twenty-one percent of the Staphylococcus spp. was methicillin resistance $S$. aureus (MRSA). There were also some multi-drugs resistance (MDR) organisms such as Acinetobacter species, which were resistant to all of the tested antibiotics (Table 2).

\section{Catheter infection and related factors}

Catheter infection was more common in men. Forty-four patients had femoral catheters, 25 of whom (71\%) were infected, 79 had jugular and 28 had subclavian catheters. Forty $(26.4 \%)$ of the jugular catheters and 15 (9.9\%) of the subclavian ones were infected (Table 3 ).

Table 3 depicts some risk factors for the CRBSIs, in which only gender of cases has a significant difference for catheter infection $(P<0.05)$. The impact of male gender on the CRBSI according to multivariate logistic regression analysis, showed odds ratio $(\mathrm{OR})=2.17$ (95\% CI, 1.11$4.21)$.

Among all patients indicated for peripheral blood and

Table 2. Organisms and antibiotic resistance in CRBSI cases

\begin{tabular}{|c|c|c|c|c|c|c|}
\hline Antibiotics & $\begin{array}{c}\text { Staphylococcus spp., } \\
\text { N (\%) }\end{array}$ & $\begin{array}{c}\text { Enterobacteriaceae, } \\
\mathrm{N}(\%)\end{array}$ & $\begin{array}{l}\text { Enterococcus } \\
\text { spp., } \mathbf{N}(\%)\end{array}$ & $\begin{array}{c}\text { Pseudomonas } \\
\text { aeruginosa, N (\%) }\end{array}$ & $\begin{array}{l}\text { Acinetobacter } \\
\text { spp., } \mathrm{N}(\%)\end{array}$ & $\begin{array}{l}\text { Stenotrophomonas } \\
\text { maltophilia, N (\%) }\end{array}$ \\
\hline Penicillin & $\begin{array}{c}S=9(16), R=47(83) \\
n=56\end{array}$ & & $\begin{array}{c}S=2(40), R=3 \\
(60), n=5\end{array}$ & & & \\
\hline Cefoxitin & $\begin{array}{c}S=45(78), R=12(21), \\
n=57\end{array}$ & & & & & \\
\hline Erythromycin & $\begin{array}{c}\mathrm{S}=15(25), \mathrm{R}=43(74), \\
\mathrm{n}=58\end{array}$ & & & & & \\
\hline $\begin{array}{l}\text { Trimethoprim/ } \\
\text { Sulfamethoxazole }\end{array}$ & $\begin{array}{c}\mathrm{S}=33(61), \mathrm{R}=21(39), \\
\mathrm{n}=54\end{array}$ & $\begin{array}{c}S=3(43), R=4(57) \\
n=7\end{array}$ & & & $\begin{array}{c}S=1(25), R=3 \\
(75), n=4\end{array}$ & $\begin{array}{c}S=2(100) \\
n=2\end{array}$ \\
\hline Clindamycin & $\begin{array}{c}S=18(33), R=40(67), \\
n=58\end{array}$ & & & & & \\
\hline Tetracycline & $\begin{array}{c}S=11(40), R=16(60), \\
n=27\end{array}$ & $S=3(43), R=4(57), n=7$ & & & $\begin{array}{c}S=1(25), R=3 \\
(75), n=4\end{array}$ & $\begin{array}{c}S=1(50), R=1(50), \\
n=2\end{array}$ \\
\hline Ciprofloxacin & $\begin{array}{c}S=16(30), R=37(70), \\
n=53\end{array}$ & $S=1(20), R=4(80), n=5$ & & $\begin{array}{c}S=1(100) \\
n=1\end{array}$ & $\begin{array}{c}S=1(25), R=3 \\
(75), n=4\end{array}$ & $\begin{array}{c}S=1(50), R=1(50), \\
n=2\end{array}$ \\
\hline Gentamycin & & $S=2(33), R=4(66), n=6$ & $S=1(100), n=1$ & $S=1(100)$ & $\begin{array}{c}S=1(25), R=3 \\
(75), n=4\end{array}$ & \\
\hline Vancomycin & & & $\begin{array}{c}R=2(40), S=3 \\
(60), n=5\end{array}$ & & & \\
\hline Amikacin & & $S=6(100), n=6$ & & $S=1(100), n=1$ & $\begin{array}{c}S=1(25), R=3 \\
(75), n=4\end{array}$ & \\
\hline Imipenem & & $S=3(75), R=1(25), n=4$ & & $\begin{array}{c}S=1(100), \\
n=1\end{array}$ & $\begin{array}{c}S=1(25), R=3 \\
(75), n=4\end{array}$ & \\
\hline Cefepime & & $R=1(100), n=1$ & & & & \\
\hline Levofloxacin & & $R=1(100), n=1$ & & & & $\begin{array}{c}S=1(50), R=1(50), \\
n=2\end{array}$ \\
\hline Cefotaxime & & & & & $\begin{array}{c}S=1(25), R=3 \\
(75), n=4\end{array}$ & \\
\hline
\end{tabular}

$S$, sensitive; $R$, resistance; $n$, number of times that antibiotic susceptibility test has been done. 
Table 3. Risk factors that are probably related to CRBSI in hemodialysis patients

\begin{tabular}{|c|c|c|c|c|}
\hline & & Positive CRBSI ( $n=84)$ & Negative CRBSI $(n=67)$ & $P$ value \\
\hline Age & Age average & $62.94 \pm 16.11$ & $57.53 \pm 19.15$ & 0.062 \\
\hline \multirow{2}{*}{ Gender } & Male & 58 (38\%) & $34(22 \%)$ & \multirow{2}{*}{0.029} \\
\hline & Female & $26(17 \%)$ & $33(21 \%)$ & \\
\hline \multirow{3}{*}{ Site of catheter } & Jugular & $40(26.4 \%)$ & $39(25.8 \%)$ & \multirow{3}{*}{0.255} \\
\hline & Subclavian & $15(9.9 \%)$ & $13(8.6 \%)$ & \\
\hline & Femoral & $29(19.2 \%)$ & 15 (9.9\%) & \\
\hline \multirow{3}{*}{ Comorbidity } & HTN & $21(14 \%)$ & $16(10 \%)$ & \multirow{3}{*}{0.736} \\
\hline & HTN, DM & $16(10 \%)$ & $11(7 \%)$ & \\
\hline & CVD, HTN & $4(2.5 \%)$ & $5(3 \%)$ & \\
\hline
\end{tabular}

HTN, hypertension; DM, diabetes mellitus; CVD, cardiovascular disease.

$T$ test and chi-square test were performed and for age and other factors, respectively.

catheter culture, approximately $52 \%$ had considerable clinical presentations of infection, which was fever or chills. Other cases had no significant fever or chills. Hypertension and diabetes were the main comorbidities in our patients. About CRBSIs, only four patients had tunnel infection and their catheters were immediately removed. Among all patients, five cases had endocarditis which $S$. aureus was the main organism cultured. All endocarditis patients had both fever and severe leukocytosis (WBC more than $20000 / \mu \mathrm{L})$.

\section{Discussion}

Hemodialysis patients in all centers are prone to CRBSIs, due to immune dysfunction and repetitive exposure to health environment (11). There are some differences between centers about incidence of CRBSI. Incidence of CRBSI, was 1.2-2.5 per 1000 catheter/days in Canadian study. In other studies 6.1 per 1000 catheter - days was reported $(1,14)$. We suppose that the difference in catheter care behaviors, type of catheters, or duration of catheter indwelling may influence on the results. Periodic and simultaneous studies should be done in each geographic area to compare the incidence of infection between centers.

CRBSIs related microorganisms and risk factors in our study are similar to other studies. In this study, the most common organisms were coagulase negative Staphylococcus species and S. aureus, respectively. These organisms are also the most common in other studies (14-17).

Conversely, S aureus-Staphylococcus coagulase negative and Enterobacter-Pseudomonas were common in another report (18).

In the majority of CRBSIs, the most common organisms are gram-positive organisms (52-84\%). Among all the Staphylococcus species, $21 \%$ had resistance to cefoxitin (second-generation cephamycin antibiotic), which indicates a high prevalence of MRSA in infected catheters. The high prevalence of MRSA in these patients may be due to their frequent visits to the hospital for their hemodialysis process and exposure to resistant organisms.
Almost in all the studies about catheter infection, MRSA infections were reported $(12,19)$.

In some centers up to $33 \%-43 \%$ of CRBSIs are MRSA, which has been associated with great cost and high mortality (20). In another report, out of 128 positive cultures, 53 were positive for $S$. aureus, which 30 of them was MRSA (21).

Considering high prevalence of MRSA in infected patients with Staphylococcus species, empiric antibiotic must be effective on these organisms. In hemodialysis centers with an elevated frequency of MRSA organisms, vancomycin is appropriate for empirical antibiotic treatment. If vancomycin minimum inhibitory concentration (MIC) is more than $12 \mathrm{mg} / \mathrm{mL}$, other alternative agents, such as daptomycin, should be selected $(12,22)$.

Among the gram-negative bacteria species, there were MDR organisms (such as A. baumannii). Considering the existence of the above mentioned organisms, appropriate antibiotics should be used. Other studies have referred to these organisms and high antibiotics resistance of them too $(12,23)$. In a study, gram-negative species were isolated in $27 \%-36 \%$ of episodes and fungi were less common $(<10 \%)$ (24).

In another study, Pseudomonas/Stenotrophomonas accounts for $4 \%-16 \%$ of microorganisms involved in CRBSIs (25).

About gram-negative bacilli microorganisms, local antibiotic susceptibility information and severity of infection are necessary for empirical therapy, while fourth generation cephalosporin, carbapenem or $\beta$-lactam/ lactamase together with or without an aminoglycoside are appropriate $(22,26,27)$.

All culture samples in our study showed only single type of organism growth. Conversely, in a study, 16-20\% of CRBSIs had multiple organisms in culture that is a challenging matter for antibiotic selection (28).

Empiric combination antibiotic therapy for MDR gramnegative bacilli (such as $P$. aeruginosa) must be used, when CRBSIs are suspected in neutropenic patients, severely ill patients with sepsis, or patients recognized to be colonized 
with such pathogens until cultures results and antibiotic susceptibility to be determined (29-31).

Our study showed 55 subjects $(65 \%)$ had fever or chills. Eighty-five percent of suspected cases of CRBSIs, which had fever or chills immediately after beginning of hemodialysis, had positive cultures. As a rule, fluctuations of temperature in hemodialysis patients are common (32). In a study, frequency of fever or chills was $53 \%$. In other studies, $44 \%$ of patients with CRBSIs had fever or chills $(18,33)$. We should differentiate original infection from others in these patients. On the other hand, in a study in Pakistan, $51 \%$ of cultures sent to laboratory were positive (34). Hence, clinicians must consider clinical symptoms or signs of inflammation in hemodialysis patients. However, clinical manifestation is not a reliable method for diagnosis of CRBSI, since confirmatory laboratory diagnosis is necessary.

In our study, male gender was a risk factor for CRBSI (Table 3). There are converse results of risk factors in multiple studies. Diabetes was identified as a risk factor in one study (17). Gender and site of catheter were evaluated in another study, which had a significant association with CRBSI (35). In a study, femoral site of catheter insertion was a risk factor for CRBSIs (36). Other factors such as type of catheter, average age and comorbidities did not have a significant association with catheter infection (18). Some studies showed that diabetes and prolonged duration of catheter were risk factors for CRBSIs $(14,37$, 38). Studies showed that previous history of CRBSIs was a risk factor $(35,39)$. The multiple analysis clearly indicated that hypertension, atherosclerosis, and site of catheter insertion (femoral versus jugular) were important risk factors for bacteremia episodes (40,41). Finally, we suppose that diversity of etiologic factors, or time for follow-up may influence on frequency of reported risk factors in centers. Although the prevalence of diabetes mellitus or hypertension is different between centers, hypertension and diabetes are the most common comorbidities in all of the patients with hemodialysis around the world like our study $(8,12)$.

\section{Conclusion}

Practical findings of this study are;

1. Concerning, the high frequency of gram positive and gram-negative microorganisms in the center, coverage of all of them is recommended for empirical treatment of CRBSIs.

2. Vancomycin is recommended for initial treatment of CRBSI to cover the MRSA organisms.

3. Male patients are more prone to CRBSIs, which mandates further preventive and therapeutic maneuvers in these groups.

The catheter infection in hemodialysis patients can result in catastrophic complications such as septicemia and endocarditis. Our recommendations to future studies include focusing on the preventive methods, i.e. reducing risk factors of the catheter infection and further strict hygienic and aseptic behaviors in dialysis centers.

However, the best way to prevent catheter infection is earlier diagnosis and referral pre-dialysis patients to a nephrologist for insertion of arteriovenous access (fistula or graft) or transplantation.

\section{Limitations of the study}

The most important limitation in our study was low number of evaluated patients compared to larger studies. On the other hand, blood cultures were performed only in cases of suspected CRBSI, so we could have missed subclinical episodes. Moreover, some of the patients (approximately 8\%) had obscure data about their catheters.

\section{Acknowledgments}

The authors would like to thank the Clinical Research Center of Lorestan University of Medical Sciences for their supports.

\section{Authors' contribution}

$\mathrm{BH}$ participated in the design and conduct of the study and preparation of the manuscript. AZM participated in describing the methodology of the study and statistical analysis of the data and amending the manuscript draft of the article. MR participated in the statistical analysis and preparation of the manuscript draft. $\mathrm{BH}$ prepared the draft of the proposal and participated in conduct of the study (data collection, import of data into the software).

\section{Conflicts of interest}

The authors declared no competing interests.

\section{Ethical considerations}

Ethical issues including plagiarism, double publication, and redundancy have been completely observed by the authors.

\section{Funding/Support}

This research was supported by Lorestan University of Medical Sciences (Grant\#1071).

\section{References}

1. Lata C, Girard L, Parkins M, James MT. Catheter-related bloodstream infection in end-stage kidney disease: a Canadian narrative review. Can J Kidney Health Dis. 2016; 3:24. doi: 10.1186/s40697-016-0115-8.

2. USRDS: US Renal Data System. USRDS 2009 Annual Data Report: Atlas of Chronic Kidney Disease and End-Stage Renal Disease in the United States, National Institutes of Health. Bethesda, MD: National Institute of Diabetes and Digestive and Kidney Diseases: 2009. p 273.

3. Zhang J, Burr RA, Sheth HS, Piraino B. Organism specific bacteremia by hemodialysis access. Clin Nephrol 2016; 86: 141-6. 
4. Suzuki M, Satoh N, Nakamura M, Horita S, Seki G, Moriya K. Bacteremia in hemodialysis patients. World J Nephrol. 2016;5:489-96. doi: 10.5527/wjn.v5.i6.489.

5. Ishani A, Collins AJ, Herzog CA, Foley RN. Septicemia, access and cardiovascular disease in dialysis patients: the USRDS Wave 2 study. Kidney Int. 2005;68:311-8.

6. Ramanathan V, Chiu EJ, Thomas JT, Khan A, Dolson GM, Darouiche RO. Healthcare costs associated with hemodialysis catheter-related infections: a single-center experience. Infect Control Hosp Epidemiol. 2007;28:606-9.

7. Gonçalves EA, Andreoli MC, Watanabe R, Freitas MC, Pedrosa AC, Manfredi SR, et al. Effect of temporary catheter and later referral on hospitalization and mortality during the first year of hemodialysis treatment. Artf Organs 2004;28:1043-9.

8. Safdar N, Mermel LA, Maki DG. The epidemiology of catheter-related infection in the critically ill. In: O'Grady NP, Pittet D, eds. Catheter-Related Infections in the Critically ill. Perspectives on Critical Care Infectious Diseases. vol 8. Springer, Boston, MA; 2004. p.1-22.

9. Schönenberger M, Forster C, Siegemund, M, Woodtli S, Dickenmann M. Catheter related blood stream infections in critically ill patients with continuous haemo (dia) filtration and temporary non-tunnelled vascular access. Swiss Med Wkly. 2011;141:47-48. doi: 10.4414/smw.2011.13294.

10. Lok CE, Mokrzycki MH. Prevention and management of catheter-related infection in hemodialysis patients. Kidney Int. 2011;79:587-98. doi:10.1038/ki.2010.471

11. CDC. Dialysis Event Surveillance Protocol. Available from: https://www.cdc.gov/nhsn/PDFs/pscManual/8pscDialysis Eventcurrent.pdf.

12. Mermel LA, Allon M, Bouza E, Craven DE, Flynn P, O'Grady $\mathrm{NP}$, et al. Clinical practice guidelines for the diagnosis and management of intravascular catheter-related infection: 2009 Update by the Infectious Diseases Society of America. Clin Infect Dis 2009;49:1-45.

13. The Clinical and Laboratory Standards Institute. Performance Standards for Antimicrobial Susceptibility Testing. 29th ed. CLSI supplement M100. Wayne, PA: CLSI; 2019. https://clsi.org/standards/products/free-resources/ access-our-free-resources.

14. Katneni R, Hedayati S. Central venous catheter-related bacteremia in chronic hemodialysis patients: epidemiology and evidence-based management. Nat Rev Nephrol 2007;3:256-66. doi: 10.1038/ncpneph0447.

15. Menegueti MG, Betoni NC, Bellissimo-Rodrigues F, Romão EA. Central venous catheter-related infections in patients receiving short-term hemodialysis therapy: incidence, associated factors, and microbiological aspects. Rev Soc Bras Med Trop. 2017;50:783-7.

16. Teo BW, Sau PY, Xu H, Ma V, Vathsala A, LeeEJ. Microbiology of tunnelled catheter-related infections in a multi-ethnic South-East Asian patient population. Nephron Clinical Practice. 2011;118:c86-c92. doi: 10.1159/000319866.

17. Nassar GM, Ayus JC. Infectious complications of the hemodialysis access. Kidney Int. 2001;60:1-13. doi: 10.1046/j.1523-1755.2001.00765.x.

18. Vercaigne LM, Gosnell T, Gin A. A retrospective analysis of catheter-related infections in a hemodialysis population. Can J Hosp Pharm. 2018;52(2). doi:10.4212/cjhp.v52i2.1780
19. Lok CE, Mokrzycki MH. Prevention and management of catheter-related infection in hemodialysis patients. Kidney Int. 2011;79:587-98. doi: 10.1038/ki.2010.471

20. Reed SD, Friedman JY, Engemann JJ, Griffiths RI, Anstrom KJ, Kaye KS, et al. Costs and outcomes among hemodialysis-dependent patients with methicillinresistant or methicillin-susceptible Staphylococcus aureus bacteremia. Infect Control Hosp Epidemiol. 2005;26:175183. doi: 10.1086/502523.

21. Grothe C, Belasco AGS, Bittencourt ARC, Vianna LAC, Sesso RCC, Barbosa DA. Incidence of Bloodstream infection among patients on hemodialysis by central venous catheter. Rev Latino-Am Enfermagem 2010;18:73-80. doi: 10.1590/s0104-11692010000100012.

22. Sakoulas G, Moise-Broder PA, Schentag J, Forrest A, Moellering RC, Eliopoulos GM. Relationship of MIC and bactericidal activity to efficacy of vancomycin for treatment of methicillin-resistant Staphylococcus aureus bacteremia. J Clin Microbiol. 2004;42:2398-402. doi: 10.1128/ JCM.42.6.2398-2402.2004.

23. Seifert H, Strate A, Pulverer G. Nosocomial bacteremia due to Acinetobacter baumannii. Clinical features, epidemiology, and predictors of mortality. Medicine. 1995; 74:340-9.

24. Mokrzycki MH, Zhang M, Cohen H, Golestaneh L, Laut JM, Rosenberg SO. Tunnelled hemodialysis catheter bacteremia: risk factors for bacteremia recurrence, infectious complications and mortality. Nephrol Dial Transplant. 2006;21:1024-31. doi: 10.1093/ndt/gfi104.

25. Golestaneh L, Laut J, Rosenberg S, Zhang M, Mokrzycki MH. Favourable outcomes in episodes of Pseudomonas bacteraemia when associated with tunneled cuffed catheters (TCCs) in chronic hemodialysis patients. Nephrol Dial Transplant. 2006;21(5):1328-1333. doi: 10.1093/ndt/ gfk078.

26. Moise PA, Sakoulas G, Forrest A, Schentag JJ. Vancomycin in vitro bactericidal activity and its relationship to efficacy in clearance of methicillin-resistant Staphylococcus aureus bacteremia. Antimicrob Agents Chemother. 2007;51:25826. doi: 10.1128/AAC.00939-06.

27. Wilcox MH, Tack KJ, Bouza E, Herr DL, Ruf BR, Ijzerman $\mathrm{MM}$, et al. Complicated skin and skin-structure infections and catheter-related bloodstream infections: noninferiority of linezolid in a phase 3 study. Clin Infect Dis. 2009; 48:20312. doi: $10.1086 / 595686$.

28. Saxena AK, Panbotra BR. Haemodialysis catheter-related bloodstream infections: current treatment options and strategies for prevention. Swiss Med Wkly. 2005;135:12738.

29. Rodríguez-Créixems M, Alcalá L, Muñoz P, Cercenado E, Vicente T, Bouza E. Bloodstream infections: evolution and trends in the microbiology workload, incidence, and etiology, 1985-2006. Medicine. 2008;87:234-49. doi: 10.1097/MD.0b013e318182119b.

30. Chee L, Brown M, Sasadeusz J, MacGregor L, Grigg A. Gram-negative organisms predominate in Hickman line-related infections in non-neutropenic patients with hematological malignancies. J Infect. 2008;56:227-33. doi: 10.1016/j.jinf.2008.01.046.

31. Blot SI, Depuydt P, Annemans L, Benoit D, Hoste E, De Waele JJ, et al. Clinical and economic outcomes in 
critically ill patients with nosocomial catheter-related bloodstream infections. Clin Infect Dis. 2005;41:1591-8. doi: $10.1086 / 497833$.

32. Daugirdas JT, Blake PG, Ing TS, eds. Handbook of Dialysis. 5th ed. Philadelphia: Wolters Kluwer Health; 2015. p. 615617.

33. Quittnat Pelletier F, Joarder M, Poutanen SM, Lok CE. Evaluating approaches for the diagnosis of hemodialysis catheter-related bloodstream infections. Clin J Am Soc Nephrol. 2016;11:847-54. doi: 10.2215/CJN.09110815.

34. Qureshi AL, Abid K. Frequency of catheter related infections in haemodialysed Uraemic patients. J Pak Med Assoc. 2010;60(8):671-5.

35. Lemaire X, Morena M, Leray-Moragués H, Henriet-Viprey D, Chenine L, Defez-Fougeron C, et al. Analysis of Risk factors for catheter- related bacteremia in 2000 permanent dual catheters for hemodialysis. Blood Purif. 2009;28:2128. doi: 10.1159/000210034.

36. Goetz AM, Wagener MM, Miller JM, Muder RR. Risk of infection due to central venous catheters: effect of site of placement and catheter type. Infect Control Hosp Epidemiol. 1998;19:842-5. doi: 10.1086/647742.

37. Oliver MJ, Callery SM, Thorpe KE, Schwab SJ, Churchill DN. Risk of bacteremia from temporary hemodialysis catheters by site of insertion and duration of use: a prospective study. Kidney Int. 2000;58:2543-5. doi: 10.1046/j.1523-1755.2000.00439.x

38. Wang K, Wang P, Liang X, Lu X, Liu Z. Epidemiology of haemodialysis catheter complications: a survey of 865 dialysis patients from 14 haemodialysis centers in Henan province in China. BMJ Open. 2015;5:e07136. doi: 10.1136/bmjopen-2014-007136

39. Hoen B, Paul-Dauphin A, Hestin D, Kessler M. EPIBACDIAL: a multicenter prospective study of risk factors for bacteremia in chronic hemodialysis patients. J Am Soc Nephrol. 1998;9:869-76.

40. Menegueti MG, Betoni NC, Bellissimo-Rodrigues F, Romão EA. Central venous catheter-related infections in patients receiving short-term hemodialysis therapy: incidence, associated factors, and microbiological aspects. Rev Soc Bras Med Trop. 2017;50:783-787. doi: 10.1590/0037-86820438-2017.

41. Sedlacek M, Gemery JM, Cheung AL, Bayer AS, Remillard BD. Aspirin treatment is associated with a significantly decreased risk of Staphylococcus aureus bacteremia in hemodialysis patients with tunneled catheters. Am J Kidney Dis. 2007;49:401-8. doi: 10.1053/j.ajkd.2006.12.014.

Copyright $\odot 2020$ The Author(s); Published by Nickan Research Institute. This is an open-access article distributed under the terms of the Creative Commons Attribution License (http://creativecommons.org/licenses/by/4.0), which permits unrestricted use, distribution, and reproduction in any medium, provided the original work is properly cited. 